\title{
El autoaprendizaje como clave para incentivar la motivación y mejorar el rendimiento académico.
}

\author{
Mapelli-Brahm, Paula ${ }^{a}$ \\ ${ }^{a}$ Laboratorio Color y Calidad de Alimentos, área de Nutrición y Bromatología, Universidad de Sevilla, 41012 Sevilla, \\ España.pmapelli@us.es
}

\section{\$EWWFW}

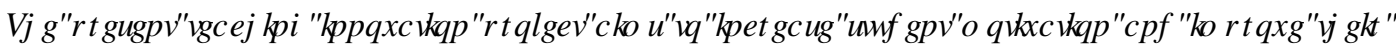

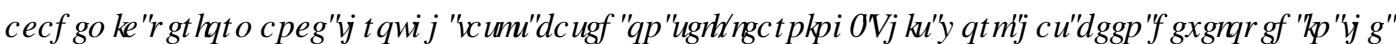

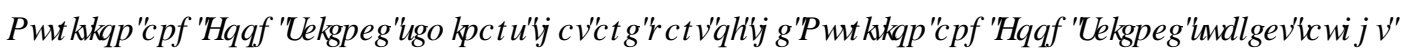

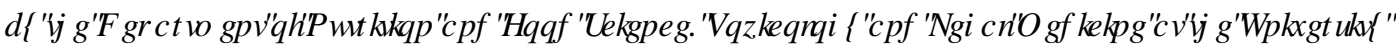

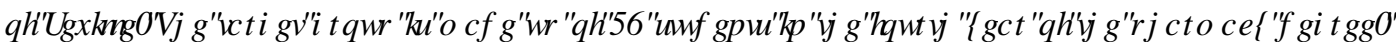

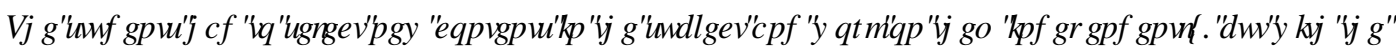

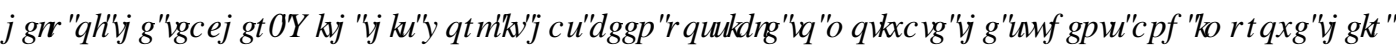

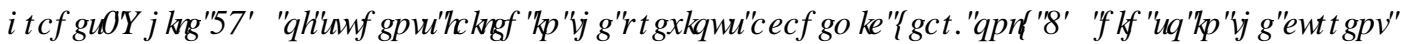

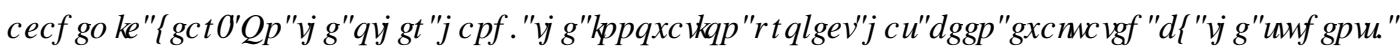

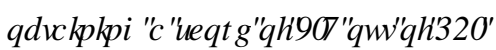

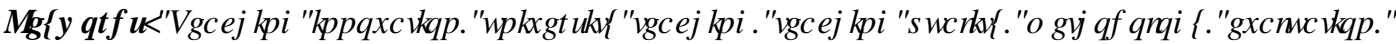
1 XWIRQDQQG\% $/ R P$ DMRQRJ।

[

\section{HXP HQ}

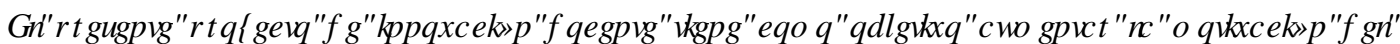

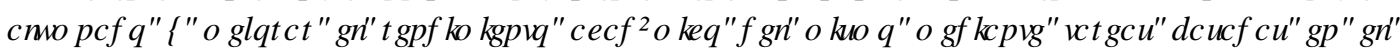

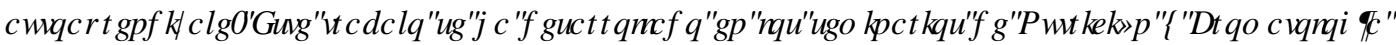

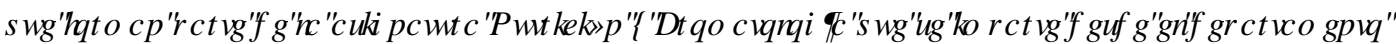

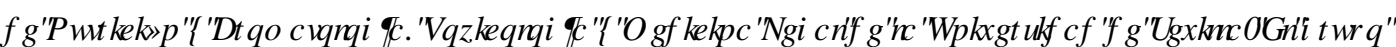

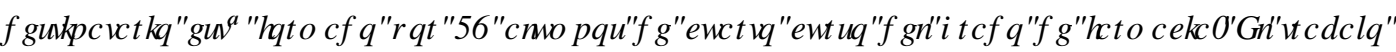

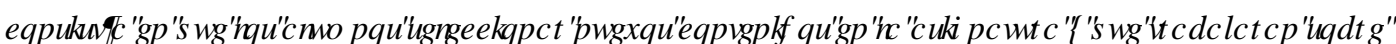

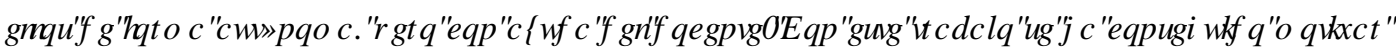

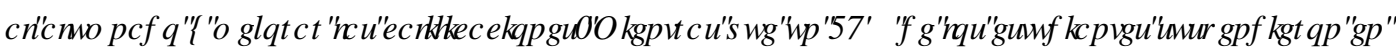

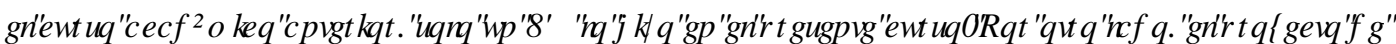

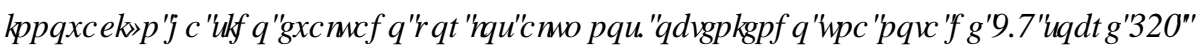

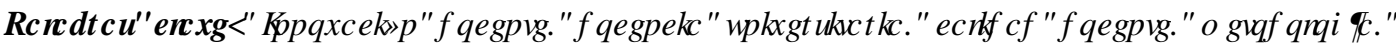

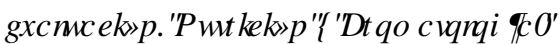

口

प 


\section{Introducción}

El presente proyecto de innovación docente universitaria se ha llevado a cabo con alumnos de cuarto curso del grado de farmacia, en los seminarios de Nutrición y Bromatología que forman parte de la asignatura Nutrición y Bromatología que se imparte desde el departamento de Nutrición y Bromatología, Toxicología y Medicina Legal de la Universidad de Sevilla. Esta asignatura es cuatrimestral y tiene 7,5 créditos. La asistencia a los seminarios no es obligatoria, a pesar de que la asignatura es de tipo obligatoria.

Por cada grupo de teoría existen varios grupos de seminarios y los profesores que imparten teoría y los que imparten seminarios están en contacto para el correcto desarrollo de la asignatura. Todos los grupos de seminarios se evalúan con un examen final común que deben aprobar para poder aprobar la asignatura completa. Por este motivo, los nueve profesores de seminario deben llegar, previo al comienzo de las clases, a un acuerdo sobre el contenido a tratar en los seminarios.

El presente proyecto se ha llevado a cabo en uno de los grupos de seminarios (34 alumnos) que conforman el grupo de teoría y solo ha participado en él el profesor responsable de este grupo, autor de este trabajo.

Normalmente, la mayoría de los alumnos suelen asistir presencialmente a los seminarios, pero, dadas las circunstancias excepcionales que vivimos estos días cómo consecuencia de la pandemia de enfermedad por coronavirus, esta asignatura se ha impartido casi en su totalidad de forma semipresencial con un escenario del $25 \%$ de presencialidad. Es decir, cada alumno asiste a una clase presencial por cada cuatro clases impartidas, mientras que el resto de las clases las sigue de forma virtual. Así, nueve alumnos como máximo pueden asistir a cada clase de forma presencial.

El desarrollo de los seminarios suele ser correcto ya que los alumnos que asisten son de cuarto curso. El número de suspensos es relativamente elevado (35\%: 12 repetidores de 34 alumnos), lo que podría afectar a la motivación del alumnado por esta asignatura. De hecho, en una investigación en la que se estudió la relación entre la motivación y el rendimiento académico se obtuvo un coeficiente de correlación de Pearson positivo $(r=0,116 ; S<0,05)$ que indicaba que estas dos variables mantenían una relación directa (Escaño y Gil de la Serna, 2008). Es decir, generalmente, cuanto mayor es la motivación del estudiante mayor será su rendimiento académico y, además, cuanto mejores son los resultados académicos, mayor será la disposición del alumnado. Son estas circunstancias las que dan sentido a este trabajo de innovación en el que se pretende, a través de una mejora en la metodología, mejorar la satisfacción del alumnado y las calificaciones. Para ello se ha tomado como principio lo indicado por Bain (2007), es decir, que el aprendizaje es más efectivo cuando los estudiantes: 1) intentan resolver problemas que consideran relevantes, intrigantes y/o motivadores; 2) son capaces de hacerlo en un entorno de apoyo, desafiante y en el que sienten que tienen el control sobre su propia educación; 3) pueden trabajar en grupos colaborativos para superar los problemas; 4) consideran que su trabajo será considerado de forma justa y honesta; y 5) tienen la oportunidad probar, errar y retroalimentarse de otros compañeros con más experiencia antes e independientemente de cualquier juicio sobre sus esfuerzos.

\section{Objetivos}

El número de suspensos en los seminarios de Nutrición y Bromatología suele ser elevado, el 35\% de los alumnos del presente curso son repetidores, y parece que existe una clara relación entre la motivación y el rendimiento académico (Escaño y Gil de la Serna, 2008). Por ello, los objetivos de este proyecto son:

- Mejorar el rendimiento académico de los alumnos.

- Incentivar el interés de los estudiantes. 
Para alcanzar estos objetivos se modificará la metodología que se usa normalmente para impartir esta asignatura y se incluirán contenidos en la misma que puedan resultar motivadores para los estudiantes. En particular, se fomentará el autoaprendizaje como base para incrementar la curiosidad, el interés y la motivación de los alumnos para, en último lugar, mejorar sus calificaciones. Con el fin de analizar si los objetivos planteados se han alcanzado, se compararán las calificaciones obtenidas en el curso académico anterior con las obtenidas el presente curso. Además, se realizará un cuestionario para evaluar la satisfacción e interés de los estudiantes.

\section{Desarrollo de la innovación}

\subsection{Contenido a trabajar}

El temario de la asignatura es el siguiente:

S1.Valoración del estado nutricional. Antropometría

S2.Evaluación de la calidad de las proteínas

S3.Metabolismo energético. Estimación gasto energético

S4.Composición de alimentos y evaluación de dietas

S5.Biodisponibilidad de nutrientes. Aporte de Hierro

S6.Legislación alimentaria

S7.Etiquetado de alimentos

S8.Análisis de nutrientes. Macronutrientes y micronutrientes

S9.Aditivos alimentarios

El tema sobre el que se ha trabajado se engloba dentro del Seminario 2: Evaluación de la calidad de las proteínas. Este tema tiene gran relevancia, atendiendo al hecho de que las proteínas están muy relacionadas con aspectos de nuestra vida diaria. Así, por ejemplo, el conocimiento sobre proteínas es fundamental para el correcto desarrollo de dietas y suplementos.

En años anteriores, este seminario (S2), estaba fundamente enfocado al estudio de la calidad de las proteínas con el objetivo fundamental de que los estudiantes aprendieran a realizar correctamente los ejercicios de cálculo planteados. La teoría no se relacionaba con aspectos cotidianos, lo que podría ser una de las causas de la desmotivación del alumnado. En el presente curso, los alumnos, guiados por el docente, han seleccionado nuevos contenidos sobre los que trabajar que pudiesen resultar motivadores para ellos por estar relacionados con aspectos de la sociedad actual. En particular, durante las clases en las que se ha desarrollado este trabajo se han tratado nuevos contenidos relacionados con las necesidades proteicas en atletas y en vegetarianos. Se incrementa así el contenido de tipo actitudinal en este seminario (Del Carmen, 1996), ya que los estudiantes pueden aprender a emitir consejos nutricionales y alimentarios a distintos grupos de población atendiendo a sus características. 


\subsection{Modelo metodológico}

Dado que es importante que el proceso educativo se ajuste al nivel competencial inicial de los alumnos, se llevó a cabo un debate inicial para poder conocer el nivel del que parten los estudiantes. De esta forma, el docente pudo diseñar estrategias didácticas y desarrollar su labor adaptándose a la realidad del grupo destinatario (Granados-Bermúdez, 2009).

Para alcanzar los objetivos planteados, se trató de buscar un tema de actualidad que pudiese ser interesante para los alumnos y sobre el cual estos debían trabajar de forma autónoma. Es decir, la metodología se basaba en el autoaprendizaje con la finalidad de captar el interés de los estudiantes y qué, desde el trabajo del propio alumno, surgiese un conocimiento profundo y duradero (Rodrigo Herrera, et al., 2011).

A pesar del papel fundamental del estudiante en este proyecto de innovación, el profesor actuó como guía en todo momento, sirviendo de apoyo y realizando preguntas sobre el tema seleccionado para que los educandos las respondan a través de la reflexión, de la propuesta de hipótesis y de la búsqueda de información.

El trabajo propuesto para los alumnos se desarrolló en grupos para que este fuese más ameno y así asegurar la participación de los mismos. Cada grupo debía presentar los resultados por escrito y en un vídeo elaborado por ellos. Así, cabe indicar que a través del desarrollo del presente trabajo de innovación docente se puede incentivar el interés y el hábito de lectura, la práctica de la expresión escrita y la capacidad de expresarse correctamente.

El docente hizo uso de clases magistrales para enseñar los conceptos más importantes y necesarios para la resolución de las preguntas planteadas. En estas clases magistrales el profesor trató de ser un experto en la materia que imparte $\mathrm{y}$, además, un buen comunicador, trabajando con presentaciones llamativas e intentando relacionar los contenidos con aspectos de la vida cotidiana del alumno. Todo ello con el fin de incentivar el interés de los estudiantes. Basándose en la teoría construccionista del andamiaje del conocimiento de Bruner (Guilar, 2009), estos contenidos se desarrollaron en orden, desde los más fáciles a los más complicados. Al alumnado se le motivó incentivando su participación de forma que la comunicación fuese bidireccional.

Una vez generado el conocimiento sobre el tema en el que se enmarca este trabajo (a través del autoaprendizaje y de clases magistrales participativas), se resolvieron ejercicios de cálculos en el aula, fomentando la participación del alumnado.

Cabe destacar también que esta experiencia se ha desarrollado atendiendo a la diversidad. Así, en el grupo destinatario había una persona extranjera con la cual el docente mantuvo una continua comunicación para facilitar su trabajo.

Como resumen, este proyecto de innovación docente se basa en el autoaprendizaje como herramienta clave para fomentar la curiosidad y aumentar la motivación del alumno, lo que puede dar como resultado una mejora del rendimiento académico. 


\subsection{Descripción de la clase}

En la Figura 1 se presenta un resumen diseño de las sesiones para el desarrollo de este trabajo y en la Tabla 1 la secuencia de actividades.

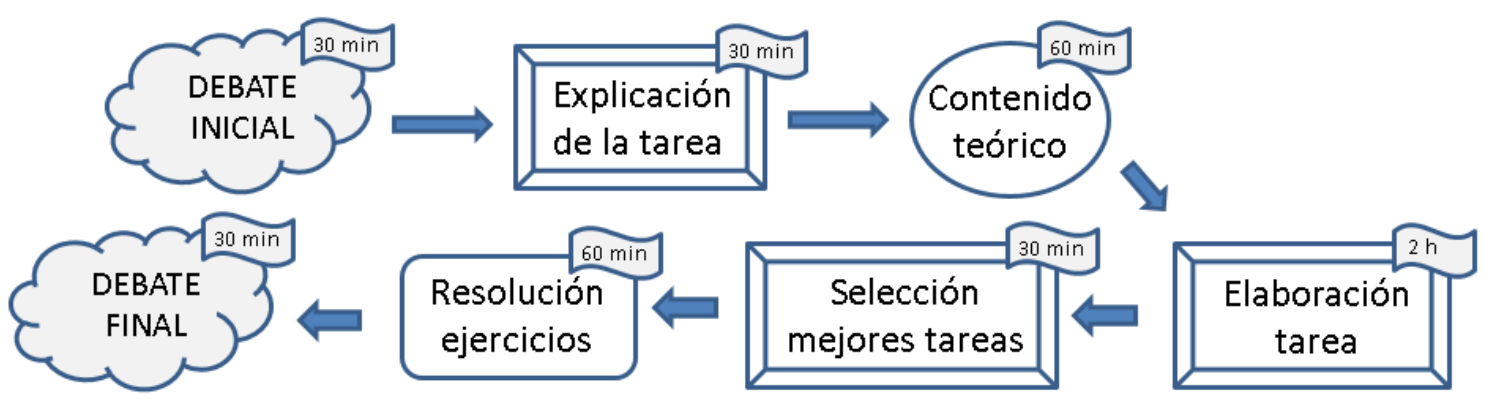

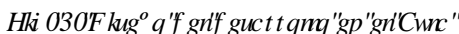

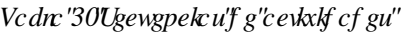

\begin{tabular}{|c|c|c|c|}
\hline Nombre & Descripción & Recursos & Duración (min) \\
\hline Debate inicial & $\begin{array}{l}\text { Se le pregunta a los alumnos cuales son los temas } \\
\text { relacionados con el contenido a tratar que les interesan }\end{array}$ & Foro E.V. & 30 \\
\hline $\begin{array}{l}\text { Explicación } \\
\text { de la tarea e } \\
\text { ideas previas. }\end{array}$ & $\begin{array}{l}\text { Se explica el trabajo a realizar y las herramientas } \\
\text { disponibles. Se exponen las ideas previas sobre la } \\
\text { tarea. }\end{array}$ & $\begin{array}{l}\text { Presentación } \\
\text { PowerPoint en clase } \\
\text { semipresencial }\end{array}$ & 30 \\
\hline $\begin{array}{l}\text { Contenido } \\
\text { teórico }\end{array}$ & Explicación teórica sobre las proteínas & $\begin{array}{l}\text { Presentación } \\
\text { PowerPoint, vídeos. } \\
\text { Foro E.V. }\end{array}$ & 60 \\
\hline $\begin{array}{l}\text { Elaboración } \\
\text { de vídeo }\end{array}$ & $\begin{array}{l}\text { Tras la lectura de ciertos artículos de investigación los } \\
\text { alumnos responden por escrito a ciertas preguntas y } \\
\text { elaboran un vídeo respondiéndolas. }\end{array}$ & $\begin{array}{l}\text { Foro E.V., } \\
\text { WhatsApp, teléfono } \\
\text { móvil, etc. }\end{array}$ & 120 \\
\hline $\begin{array}{l}\text { Selección de } \\
\text { los mejores } \\
\text { vídeos }\end{array}$ & Los alumnos deben decidir el mejor vídeo. & Padlet & 30 \\
\hline $\begin{array}{l}\text { Resolución } \\
\text { ejercicios }\end{array}$ & $\begin{array}{l}\text { Resolución de ejercicios relacionados con el tema } \\
\text { abordado }\end{array}$ & $\begin{array}{l}\text { Foro E.V., pizarra } \\
\text { clásica }\end{array}$ & 60 \\
\hline $\begin{array}{l}\text { Debate final y } \\
\text { cuestionario }\end{array}$ & $\begin{array}{l}\text { Se les pregunta a los alumnos qué les ha parecido la } \\
\text { tarea y qué han sacado en conclusión. }\end{array}$ & Foro E.V. & 30 \\
\hline
\end{tabular}

Para comenzar el debate inicial, el docente realizó algunas preguntas relacionadas con el contenido del seminario para conocer el nivel inicial de los estudiantes. Estas preguntas debían responderlas los alumnos 
de forma voluntaria, pero solo algunos participaron activamente. La baja participación podría ser debido a la poca motivación del alumnado por esta asignatura. El contenido del seminario implicaba el uso de conceptos que el alumnado usa comúnmente en su día a día, por lo que algunos estudiantes tenían ideas previas que además de ser erróneas, podrían resultar difíciles de eliminar por estar muy arraigadas.

La tarea a realizar por los alumnos se diseñó teniendo en cuenta que temas, relacionados con el contenido de la asignatura, podrían resultar más interesantes para ellos. Por ello, se pidió a los alumnos que indicaran sobre qué temas les gustaría profundizar. Se plantearon preguntas algo provocativas que despertaron el interés del alumnado. Este debate inicial duró aproximadamente $30 \mathrm{~min}$. Los temas que se plantearon fueron similares a los que el docente tenía pensado previamente, por lo que este tuvo la oportunidad de enseñar algunos recortes de revistas, periódicos, etc. que había seleccionado previamente y que mostraban la importancia que tiene para la sociedad los temas que habían sido seleccionados. De esta forma, se pretendía motivarlos para realizar la tarea.

Una vez diseñada la tarea, se explicó en qué consistía la misma: los alumnos debían responder a ciertas preguntas planteadas por el docente y relacionadas con los temas que los estudiantes habían previamente seleccionado:

1. ¿Se deberían recomendar suplementos proteicos para atletas de élite?

2. ¿Se deberían recomendar suplementos proteicos para personas que realizan un ejercicio moderado en el gimnasio?

3. ¿Podría una persona que realiza un ejercicio moderado obtener la cantidad de proteínas que necesita a través de la dieta? Comenta la importancia de la complementación proteica.

4. ¿La dieta vegetariana representa algún peligro en relación a la ingesta de proteínas?

Para responder a estas preguntas, los estudiantes debían entregar un documento con las respuestas y elaborar un pequeño vídeo de no más de 8 minutos ( 2 minutos/pregunta). El docente indicó que para poder responder a las preguntas solo se podía hacer uso de artículos científicos, tesis doctorales, etc. y que siempre se debía indicar la fuente de donde se extraía la información. El docente actuó de guía en todo momento y facilitó una serie de artículos de donde podían obtener información.

Los alumnos disponían de siete días para realizar el trabajo, que era opcional y podía realizarse en parejas (se les permitió que ellos eligieran las parejas para realizarla). Se indicó que a los que participasen y realizasen el trabajo adecuadamente se les subiría hasta 0.5 puntos si no llegaban al aprobado en el examen final.

A continuación, se les dejó unos minutos para que pensaran en las respuestas y se permitió que algún alumno responda a las mismas. Se les motivó diciendo que podrían saber las respuestas correctas al finalizar la tarea. 
A continuación, el docente procedió a explicar los contenidos teóricos más importantes (introducción a las proteínas, calidad proteica e importancia de las proteínas en nuestra dieta) a través de clases magistrales. En estas clases magistrales se buscó la participación de los alumnos. Para ello, por ejemplo, se plantearon preguntas durante la clase y se motivó a los estudiantes para que pregunten.

Una vez realizada la tarea propuesta para los estudiantes, el docente compartió los vídeos a los alumnos, para que estos eligieran la mejor respuesta entre las planteadas por los compañeros. Mediante la realización de la tarea y la selección del mejor vídeo, se pretendía que el alumnado aprenda a procesar, jerarquizar y relacionar información y a buscar estrategias de resolución.

Una vez generado el conocimiento sobre el tema en el que se enmarca este trabajo (a través del autoaprendizaje y de clases magistrales participativas), se resolvieron ejercicios de cálculos en el aula, fomentando la participación del alumnado.

Tras la selección de los mejores trabajos se desarrolló una puesta en común en la que los alumnos pudieron criticar y enriquecerse de los trabajos de sus compañeros (debate final).

Para finalizar, se pasó un cuestionario para obtener información sobre el grado de satisfacción del alumno con el proyecto. Se les pidió que valorasen del 1 al 10 de forma anónima la metodología empleada en las sesiones relativas a este trabajo de innovación.

\section{Resultados}

En general el clima de trabajo ha sido bastante bueno, aunque algunos alumnos han sido muy poco participativos. Son varios los resultados de este proyecto que indican que los objetivos planteados han sido claramente alcanzados:

En primer lugar, se alcanzó un elevado número de participantes en la tarea voluntaria propuesta. Así, un $76 \%$ de los estudiantes (26 de 34 ) realizaron esta tarea. De hecho, este número fue superior al esperado, teniendo en cuenta que la nota del trabajo solo se vería reflejada en la nota final de la asignatura para aquellos alumnos que estuviesen cercanos al cinco, pero que no hubiesen aprobado.

Por otro lado, durante la sesión en la que se explicó el contenido teórico, se observó una mayor intervención de los alumnos en comparación con las clases anteriores y durante el debate final hubo una mayor participación en comparación con el debate final. Estos hechos podrían ser debido a dos motivos principalmente. Uno de ellos es que el docente se esforzó por alejarse de la típica clase magistral e intentó motivar a los alumnos para que participen en la clase y se generaran así micro-debates. Además, parece que el tema elegido para el trabajo grupal les resultó interesante a los alumnos y les motivó a atender más en clases.

De los vídeos y textos elaborados por los alumnos se concluye que esta tarea les ha resultado interesante, pues se han esforzado en realizar un trabajo completo y de calidad. 
Además, los alumnos valoraron con un 7,5 el proyecto de innovación docente lo que muestra el elevado grado de satisfacción de los estudiantes.

Por último, un dato que muestra claramente la consecución de los objetivos es la disminución en el porcentaje de suspensos en la asignatura. Así, el año pasado un 35\% de los estudiantes suspendieron mientras que en el presente curso solo lo hizo un 6\% (Figura 2). Cabe destacar que no solo ha mejorado el porcentaje de aprobados, sino que también se ha observado una mejora de las notas en general. Así, el porcentaje de sobresalientes (9-10 sobre 10) y notables (7-8 sobre 10$)$ ha aumentado del $0 \%$ y $12 \%$ en el curso anterior al $4 \%$ y $29 \%$ en el presente curso, respectivamente. Es decir, el número de notables en el presente curso ha sido más del doble que el obtenido en el curso anterior.

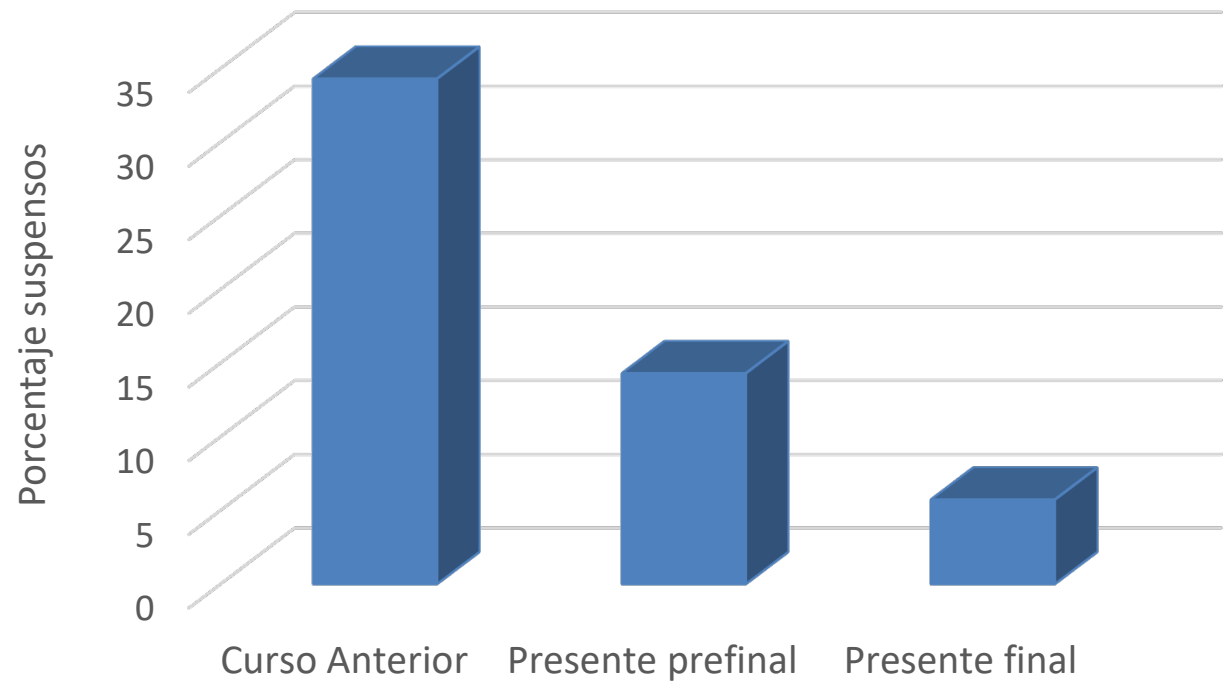

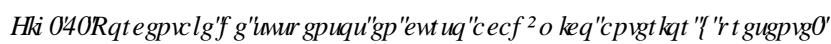

Es inevitable comentar que la experimentación del trabajo se ha visto afectada en varias ocasiones por los problemas técnicos y otros tipos de problemas derivados de la situación que vivimos actualmente.

\section{Conclusiones}

Los resultados generales de este trabajo han sido bastante satisfactorios, tanto para el alumnado como para el docente. Con el desarrollo de este trabajo la percepción de los alumnos ha mejorado y se han encontrado posibles mejoras para realizar otros trabajos similares en el futuro. Por ejemplo, a pesar del intento de enlazar unos temas con otros, se debería haber hecho un mayor hincapié en este aspecto para que los alumnos consigan tener una idea global de la asignatura y que está cobre así más sentido.

El tema elegido para el trabajo grupal ha sido un acierto, pues se ha visto bastante motivación por parte de los alumnos. Además de ser interesante para ellos, se trata de un tema de actualidad que está relacionado 
con el contenido de la asignatura. Para trabajos futuros, se le debería dar un peso importante a la búsqueda de los temas en los que centrarse, siempre completando los objetivos sobre los contenidos de la asignatura, pero buscando captar la atención del alumnado.

Atendiendo a los buenos resultados obtenidos, se espera que en un futuro este proyecto se implante en todos los grupos de seminarios, si todo el profesorado lo estima oportuno. Entre los benefícios que podría resultar de su implantación se encuentra una posible mejora de la motivación del alumnado y de su rendimiento académico. Además, con este tipo de actividades el alumnado tiene la posibilidad de tener un mayor contacto con sus compañeros lo que puede resultar también motivador. Por otro lado, este proyecto permite un mayor contacto del profesor con los alumnos, lo que puede permitir al docente conocerlos mejor y poder así adaptar su actividad docente al grupo destinatario.

\section{Referencias}

BAIN, K. (2007). / RITXHIKDFHURRVP HRLHWSLRIHRLHMXQYYHUWUIRV Valencia: Publicacions de la Universitat de Valéncia.

DEL CARMEN, L. (1996). ( ODQDOMVI UVFXHQFIDFRQQHIRVIFRQHQIGRVHCXFDUMRV. Barcelona: Horsori.

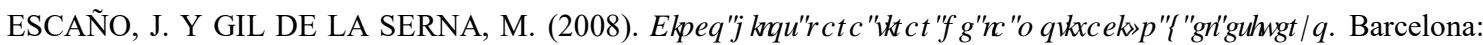
Horsori.

GUILAR, M. E. (2009).匹as ideas de Bruner: "de la revolución cognitiva" a la "revolución cultural" en ( GXFHH vol. 13, núm. 44, p. 235-241.

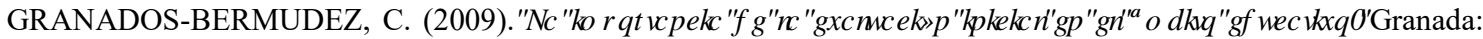
Innovación y experiencias educativas.

RODRIGO HERRERA, R.. et al. (2011). "Implementación y evaluación de una metodología de autoaprendizaje

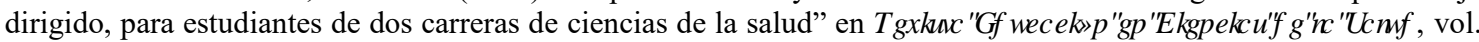
8, núm. 1, p. 25-30. 